\title{
SIMULACIÓN CLÍNICA: OPINIÓN DE ESTUDIANTES DE ENFERMERÍA， UNIVERSIDAD AUTÓNOMA DE CHILE, TEMUCO
}

\section{CLINICAL SIMULATION: NURSING STUDENT'S OPINIONS, AUTONOMOUS UNIVERSITY OF CHILE, TEMUCO}

\section{SIMULAÇÃO CLÍNICA: OPINIÃO DE ESTUDANTES DE ENFERMAGEM, UNIVERSIDAD AUTÓNOMA DO CHILE, TEMUCO}

\author{
Mónica Illesca Pretty \\ Universidad de La Frontera. Temuco, Chile \\ ORCID: 0000-0003-0635-5331 \\ monica.illesca@ufrontera.cl
}

\section{Roxana Novoa Moreno}

Universidad Autónoma de Chile. Temuco, Chile.

ORCID: 0000-0003-2014-9622

\section{Mirtha Cabezas González}

Universidad de La Frontera. Temuco, Chile

ORCID: 0000-0002-3668-8180

\author{
Alejandro Hernández Díaz \\ Escuela de Enfermería, Facultad de Ciencias, Universidad Mayor, Chile \\ ORCID: 0000-0003-3380-2846
}

\section{Luis González Osorio}

Universidad de La Frontera. Temuco, Chile

ORCID: 0000-0003-2881-5562

DOI: https://doi.org/10.22235/ech.v8i2.1845

Recibido: 04/03/2019

Aceptado: 04/07/2019

\section{RESUMEN}

La simulación clínica integra la complejidad del aprendizaje práctico-teórico con posibilidad de repetición, retroalimentación, evaluación y reflexión para lograr competencias disciplinares y genéricas del educando. El objetivo es develar la opinión de estudiantes de enfermería acerca del uso de la simulación clínica como estrategia de aprendizaje. Se trata de una investigación cualitativa exploratoria y descriptiva, mediante estudio intrínseco de casos, muestra no probabilística, intencionada de casos por criterios y conveniencia, conformada por quince informantes en el año 2016. Información recogida mediante grupos focales, analizados por reducción de datos en tres niveles, 
siguiendo el esquema de comparación constante, no agrupándolas en categorías predeterminadas, por proceso de razonamiento inductivo, validados con criterios de rigor y triangulación de investigadores. En cuanto a los resultados, en el primer nivel emergen 320 unidades de significado agrupadas en seis categorías descriptivas. En el segundo nivel hay tres metacategorías y en el tercero, dos dominios cualitativos: aportes de estudiantes para la implementación de Simulación Clínica y contribución de ésta como estrategia de aprendizaje en el desarrollo de competencias disciplinares y genéricas. Se concluye que esta metodología reduce la brecha existente entre la teoría y práctica, fortalece las competencias genéricas y permite que el estudiante asuma la responsabilidad en su formación.

Palabras claves: Simulación, Estudiantes de Enfermería, Educación Profesional, Educación en Enfermería

\begin{abstract}
The clinical simulation integrates the complexity of practical-theoretical learning with the possibility of repetition, feedback, evaluation and reflection to achieve disciplinary and generic competences of the learner. The goal is to reveal the opinion of nursing students about the use of clinical simulation as a learning strategy. It is an exploratory and descriptive qualitative research, through intrinsic case study, non-probabilistic, intentional sample of cases by criteria and convenience, made up of 15 informants in 2016. The information was collected through focus groups, analyzed by data reduction in three levels, using the constant comparison scheme, not grouping them into predetermined categories, by inductive reasoning process, validated with criteria of rigor and triangulation of researchers. In the first level 320 units of meaning emerged, grouped in six descriptive categories. In the second level there are three metacategories, in the third level there are two qualitative domains: contributions of students for the implementation of Clinical Simulation, and its contribution as a learning strategy in the development of disciplinary and generic competences. The conclusion is that this methodology reduces the existing gap between theory and practice, strengthens generic competences and allows the student to take responsibility for their training.
\end{abstract}

Keywords: Simulation, Students nursing, Education professional, Nursing Education

\title{
RESUMO
}

A simulação clínica integra a complexidade da aprendizagem teórico-prática com a possibilidade de repetição, feedback, avaliação e reflexão para obter competências disciplinares e genéricas do aprendente. Objetivo de revelar a opinião de estudantes de enfermagem sobre o uso da simulação clínica como estratégia de aprendizagem. Metodologia, pesquisa qualitativa exploratória e descritiva, por meio de estudo de caso intrínseco, não probabilístico, amostra intencional de casos por critérios e conveniência, composta por 15 informantes, 2016, informações coletadas por grupos focais, analisadas por redução de dados em três níveis, seguindo a esquema de comparação constante, não agrupando-os em categorias predeterminadas, pelo processo de raciocínio indutivo, validadas com critérios de rigor e triangulação de pesquisadores. Resultados Primeiro nível: emergem 320 unidades de significado agrupadas em 6 categorias descritivas, segundo nível: três metacategorias, domínios qualitativos do segundo nível 2: 
contribuições dos alunos para a implementação da Simulação Clínica e sua contribuição como estratégia de aprendizagem no desenvolvimento de competências disciplinares e genérico. Conclusão Esta metodologia reduz a lacuna existente entre a teoria e a prática, fortalece as competências genéricas e permite que o aluno assuma a responsabilidade pela sua formação.

Palavras chaves: Simulação, Estudantes de Enfermagem, Educação Profissionalizante, Educação em Enfermagem

\section{INTRODUCCIÓN}

La formación del estudiante de enfermería es un proceso complejo e integral que requiere la incorporación de competencias genéricas (instrumentales, sistémicas e interpersonales) y específicas, es decir, conocimientos, habilidades, actitudes y valores, junto con estrategias que permitan una evaluación integral de las competencias adquiridas. El fin último es brindar a los usuarios cuidados humanistas eficientes centrados en el logro de resultados esperados mediante la aplicación del proceso enfermero (1-3). Al revisar la historia del proceso enseñanza-aprendizaje en esta carrera, la demostración y devolución de procedimientos aparece desde sus inicios, donde los educandos aprendían entre ellos mismos algunas técnicas para desarrollar las destrezas antes de atender a los pacientes, todas con la intención de preservar uno de los principios fundamentales de la profesión que es la seguridad del paciente (4).

Hoy en día, con tecnologías avanzadas, se utiliza la simulación clínica, permitiendo la interacción de conocimientos, habilidades y factores humanos con el fin de proporcionar un entrenamiento efectivo para lograr que el alumnado desarrolle aptitudes para alcanzar las competencias, otorgando la oportunidad de una práctica análoga a la que desarrollará en la realidad asistencial (5). El aprendizaje a través de la simulación clínica otorga una respuesta clara a la necesidad de proteger la seguridad del paciente, jugando un papel muy importante a favor del principio ético de "no maleficencia", resguardando los derechos y deberes del usuario, ya que no se realizan sobre pacientes reales ni en estado crítico $(6,7)$.

La simulación es un proceso dinámico que involucra la creación de una oportunidad hipotética que incorpora una representación auténtica de la realidad, facilita la participación activa y compromiso del estudiante e integra la complejidad del aprendizaje práctico y teórico con posibilidad de repetición, retroalimentación, evaluación y reflexión (8). También se define como la recreación de un escenario ideado para experimentar la representación de un acontecimiento real con la finalidad de practicar, aprender, evaluar, probar o adquirir conocimientos de sistemas o actuaciones humanas, o cualquier actividad docente que utilice la ayuda de simuladores con el fin de estimular y favorecer el aprendizaje imitando en lo posible un escenario clínico más o menos complejo (9-11). Lo anterior da respuesta al concepto de aprendizaje significativo y el basado en la experiencia, en quienes se apoya el modelo educativo centrado en el estudiante, considerando además que la motivación de éste, durante su formación académica, es la aplicación práctica del objeto de estudio $(12,13)$. Esto demanda al docente la adquisición de nuevas competencias para llevar a cabo esta metodología, tales como el diseño de escenarios, la adecuada ejecución y su evaluación, bajo una 
concepción de aprendizaje que pone en el centro la actividad del discente (14). El aprendizaje con simulación se puede dividir en tres niveles de dificultad: baja fidelidad, realizada en modelos que permiten practicar técnicas básicas; intermedia, que agrupa habilidades que requieren un nivel de integración entre sí y alta fidelidad, que utiliza tecnologías interactivas que representan la realidad (15). Esto implica diseñar entornos adecuados, convirtiéndose en herramienta con un gran potencial docente (16).

La evaluación de las competencias del estudiante por esta vía es continua mediante una sesión con escenarios desconocidos por él, que posibilita la valoración en tres vertientes: observación del instructor, de un grupo de alumnos y la autoevaluación (4). Lo más destacado de la enseñanza por simulación es el debriefing, que consiste en la revisión autocrítica de todas las actuaciones realizadas durante una experiencia clínica, dirigida por un facultativo. También es expresado como la conversación entre varias personas para revisar un evento real o simulado, en la que los participantes analizan sus acciones y reflexionan sobre el papel de los procesos de pensamiento, las destrezas psicomotrices y los estados emocionales para mejorar o mantener su rendimiento en el futuro $(17,18)$. De ahí la importancia del debriefing para que el educando, además de desarrollar competencias específicas de la profesión, adquiera habilidades genéricas, entre ellas, autocrítica, aprender a aprender, pensamiento crítico, capacidad de análisis, entre otras.

La simulación en general, y la alta fidelidad en particular, aunque conlleva el empleo de numerosos recursos, goza de gran aceptación entre estudiantes e instructores, cumple todas las fases de la adquisición de experiencia y es la opción para la práctica de las habilidades necesarias ante situaciones críticas. Diversas publicaciones acreditan la relevancia de la simulación clínica en el proceso de formación. Muchos de ellos destacan la adquisición de competencias genéricas: mayor autonomía, autoeficacia y locus de control interno, confianza, trabajo en equipo, comunicación, mejora significativa en las destrezas interpersonales de comunicación, entrevistas y consejería, aumenta las oportunidades para la reflexión y se adquiere autoconfianza posterior a la experiencia de simulación clínica $(5,19-23)$.

En relación a lo disciplinar, los estudiantes logran un aprendizaje significativo, aplican e integran en la experiencia clínica los conocimientos adquiridos, asocian la tarea con vivencias previas, usan información sensorial, prueban técnicas propias realizadas de manera práctica reflejándose en una acción que permite efectivo entrenamiento previo a los cuidados clínicos $(19,2,22,5)$. A su vez, posibilita la realización de ensayos clínicos en el ámbito de las prácticas seguras, ejecución de procedimientos diagnósticos terapéuticos invasivos y complejos de enfermería, amén del desarrollo de actitudes como la responsabilidad profesional e interdisciplinar en la atención al paciente, a la familia y a la comunidad (4). Esta estrategia metodológica, la simulación clínica, requiere que en el curriculum se implementen protocolos que garanticen la reproducción de escenarios y se incorpore a lo largo de la carrera de forma paulatina; así el estudiante se familiariza con ellas, ya que el enfrentamiento a tecnologías desconocidas puede influir en el logro de la habilidad clínica. Es importante que haya una adecuada formación de los docentes, que sea lo más parecida a la realidad, un número suficiente de prácticas de la técnica que es necesario aprender, con la correspondientes pautas de evaluación de simulación clínica $(19,20,24)$. En este sentido es importante aclarar que la clave del éxito no 
consiste solo en tener equipos y modelos de alta tecnología, sino más bien lograr que los docentes se capaciten en esta estrategia pedagógica poniendo en práctica un modelo para desarrollar juicio clínico, salvaguardando una estrecha colaboración entre el profesorado y los estudiantes $(4,25)$. Como una forma de maximizar las vivencias, es necesario crear una cultura de la simulación clínica, así como también planificar y organizarlas de modo que guíen las actividades del plan de estudio, de acuerdo con el nivel de complejidad que se encuentra el aprendiz (4).

Así mismo, la evaluación constituye una herramienta fundamental, porque permite la rectificación de errores y aumenta el grado de realismo en la escenificación de los casos. Sin embargo, existe evidencia que los discípulos expresan su disconformidad sugiriendo una mejora en la adquisición de material real y menos simulado, ya que realizar ciertas técnicas de enfermería (gasometrías arteriales, canalización de catéteres centrales de inserción periférica, etc.) en el maniquí resulta a veces poco creíble a pesar de lo avanzado de los simuladores (5). También se han realizado trabajos en el ámbito de la pediatría, donde los estudiantes de enfermería expresan que aumenta la autoeficacia y reduce el nivel de ansiedad, adquieren mayor confianza, demostrando como consecuencia que los puntajes más altos de autoconfianza representan un aumento en la calidad de la atención de los pacientes $(26,27)$.

En este contexto, la Facultad de Ciencias de la Salud de la Universidad Autónoma, Temuco, Chile, realizó una capacitación en julio de 2016 en Simulación Clínica a docentes del área Pediátrica de la Carrera de Enfermería, con el fin de incluirla en el segundo semestre del mismo año, que fue incorporada en el Laboratorio de Enfermería Médica Pediátrica y del Adolescente. Tratándose de una primera experiencia y con el propósito de contribuir en la implementación de esta estrategia en el proceso de formación, surge la necesidad de develar la opinión de los estudiantes acerca de lo vivido con la Simulación Clínica. Los objetivos se orientan a explorar el significado, los factores que inciden, lo que han sentido al vivenciarla y la adquisición de competencias genéricas y el proceso enfermero como competencia disciplinar.

\section{METODOLOGÍA}

Se opta por el paradigma de investigación cualitativa exploratoria y descriptiva, desde la perspectiva metodológica de un estudio intrínseco de casos, cuyo fin está encaminado a describir aspectos importantes de un fenómeno poco conocido y documentarlo (28).

La experiencia se realizó con 90 estudiantes de cuarto año que cursaban la asignatura Laboratorio de Enfermería Médica Pediátrica y del Adolescente, impartida en el segundo semestre del año 2016. Se organizaron actividades de simulación clínica para que todos los estudiantes adquirieran las destrezas antes de atender a los pacientes en la práctica asistencial. Se realizaron dos actividades de baja dificultad de una hora de duración orientadas al control de signos vitales-antropometría e ingreso en pacientes pediátricos y cuatro de mediana fidelidad, de dos horas cada una, diseñadas para la gestión del cuidado en paciente pediátrico con Sepsis, Infección del Tracto Urinario, Virosis Respiratoria, Depresión Respiratoria. Las situaciones estaban programadas de manera de evaluar las competencias de saber, saber hacer y saber ser. Posterior a la ejecución de 
dichas actividades se procedió al proceso de retroalimentación con pauta específica para ello. Participaron dos docentes, con capacitación previa.

La muestra, no probabilística, intencionada de casos por criterio y por conveniencia, se conformó con 15 participantes, cuyos criterios de inclusión fueron: estudiantes de cuarto año en 2016 de la Carrera de Enfermería de la Universidad Autónoma de Chile, inscritos en primera oportunidad en el Laboratorio de Enfermería Médica Pediátrica y del Adolescente, con disponibilidad horaria y aceptación de participar en forma voluntaria previa firma del Consentimiento Informado (29).

Para la recolección de datos se utilizó la técnica de grupos focales (un grupo de 7 y otro de 8 integrantes), los que se recopilaron hasta llegar al punto de saturación, es decir, por repetición de ideas; o bien, reunir pruebas y evidencias suficientes para garantizar la credibilidad de la investigación (30). Se utilizó una pauta guía para asegurar que todos los temas fueran explorados y se empleó grabadora de audio y notas de campo para recoger toda la información, con la correspondiente autorización. El análisis de datos siguió el esquema de comparación constante, no agrupándose en categorías predeterminadas, sino más bien emergiendo de un proceso de razonamiento inductivo, a través de un método generativo y constructivo en el que se combinó la codificación inductiva de categorías con la comparación constante de ellas (31). En forma progresiva se realizó la reducción de la información concordante con el objeto de estudio a través de tres fases que conformaron diferentes tipos de operaciones, segmentación y codificación de unidades de significado, identificación de los temas principales o núcleos temáticos emergentes e integración e interpretación de los resultados en dominios cualitativos, estructurada a través de tres niveles, el que se realizó de forma manual por parte de los investigadores $(32,33)$ :

- Nivel 1: Identificación de unidades de significado y segmentación de las mismas para su agrupación en categorías descriptivas.

- Nivel 2: A partir de las categorías descriptivas se construyó un sistema de núcleos temáticos emergentes o metacategorías.

- Nivel 3: Como consecuencia del nivel anterior, se identificaron los dominios cualitativos a través de un análisis secuencial y transversal de las metacategorías.

Cabe señalar que bajo este paradigma la recolección de datos y su análisis fue concurrente (34). La categorización y segmentación fueron dos operaciones que se ejecutaron simultáneamente porque el criterio usado fue la pertenencia a un determinado concepto o tópico, donde las unidades que hacían referencia a determinada idea se incluyeron en las categorías que eran correspondientes.

La rigurosidad científica estuvo determinada por cuatro criterios: valor de verdad o credibilidad (triangulación por investigador, comprobación con participantes del estudio), aplicabilidad o transferibilidad (recogida abundante de información y descripción minuciosa), consistencia o dependencia (réplica paso a paso), y neutralidad o confirmabilidad (consenso con otros investigadores, juicio crítico de experto) (35). La validación, se llevó a cabo mediante la técnica de triangulación por investigador (28). 
Las consideraciones éticas estuvieron resguardadas desde el momento que fue un Proyecto autorizado por el Comité de Ética de la Universidad Autónoma de Chile (36). Se trabajó con los principios éticos en investigación: el valor social queda implícito en que los resultados permitirán probables beneficios sociales y científicos; la validez científica mediante un diseño riguroso, cuyos productos además fueron triangulados por investigadores, sin la participación de enfermeras docentes involucradas en la asignatura donde se realizó esta investigación; la selección equitativa de sujetos se resguardó identificando a los informantes claves, es decir, aquellos que aportaron al estudio ya que se relacionaban directamente con ésta; la proporción favorable de riesgo-beneficio enfocado en minimizar los riesgos potenciales y aumentar los beneficios en forma proporcional se cumplió respetando los principios de no-maleficencia y beneficencia, sin conflicto alguno para los participantes; la evaluación independiente hace referencia a la no distorsión de la investigación producto de los intereses de los autores, lo que se avaló por el Comité de Ética de la Universidad; el consentimiento informado, sancionado por dicho Comité que incluyó respeto a una participación voluntaria y consciente de los sujetos, ofreciéndoles la oportunidad de plantear preguntas, dudas y retirarse del estudio si lo deseaban; el respeto a los sujetos inscritos mediante la posibilidad de cambiar de opinión, asegurándoles confidencialidad de los datos y entrega de los resultados del estudio (37).

\section{RESULTADOS}

Nivel 1: Se develaron 320 unidades de significado relevantes para el estudio, agrupadas en 6 categorías emergentes, las que se puede observar en la tabla 1.

Tabla 1. Distribución porcentual de las Categorías Emergentes codificadas

\begin{tabular}{|l|l|l|l|l|}
\hline \multicolumn{2}{|l|}{} & \multicolumn{2}{l|}{$\begin{array}{l}\text { Frecuencia } \\
\text { unidades significado }\end{array}$} \\
\hline $\mathbf{N}^{\mathbf{0}}$ & Código & Categorías descriptivas codificadas & n & $\%$ \\
\hline 1 & SEX & $\begin{array}{l}\text { Significado de haber vivido esta experiencia de } \\
\text { aprendizaje }\end{array}$ & 87 & 27.2 \\
\hline 2 & SSC & Significado de Simulación Clínica & 80 & 25.0 \\
\hline 3 & CGSC & Competencias genéricas y Simulación Clínica & 42 & 13.1 \\
\hline 4 & FFSC & $\begin{array}{l}\text { Factores que favorecieron aprender en la Simulación } \\
\text { Clínica }\end{array}$ & 40 & 12.5 \\
\hline 5 & PESC & Proceso Enfermero y Simulación Clínica & 39 & 12.2 \\
\hline 6 & FDSC & $\begin{array}{l}\text { Factores que dificultan el aprendizaje con la Simulación } \\
\text { Clínica }\end{array}$ & 32 & 10.0 \\
\hline
\end{tabular}

Fuente: Elaboración propia (2018)

Como resultado de este proceso, se presenta en la Tabla 2 cada una de las seis categorías emergentes. 
Tabla 2. Distribución de frecuencias de las unidades de significado de las seis categorías emergentes

\begin{tabular}{|c|c|c|c|}
\hline Código & Categoría & \multicolumn{2}{|c|}{$\begin{array}{l}\text { Frecuencia } \\
\text { unidades } \\
\text { significado }\end{array}$} \\
\hline \multicolumn{4}{|c|}{ "Significado de haber vivido esta experiencia de aprendizaje (SEX) } \\
\hline \multirow{7}{*}{ Aspecto profesional } & $\begin{array}{l}\text { Administración/gestión (ingresos, traslados, fichas, } \\
\text { tarjetero, servicio, entrega de turno) }\end{array}$ & 15 & 17.2 \\
\hline & Técnicas y procedimientos & 14 & 16.1 \\
\hline & Conocimientos & 10 & 11.5 \\
\hline & Comunicación con equipo de salud & 7 & 8.1 \\
\hline & Trabajo en equipo & 6 & 7.0 \\
\hline & Comunicación con la familia & 4 & 4.6 \\
\hline & Educación & 1 & 1.1 \\
\hline \multirow{9}{*}{ Aspecto personal } & Seguridad & 9 & 10.3 \\
\hline & Empoderamiento del rol & 6 & 7.0 \\
\hline & Control de estrés & 5 & 5.7 \\
\hline & Organización & 3 & 3.4 \\
\hline & Tranquilidad & 2 & 2.3 \\
\hline & Crítico & 2 & 2.3 \\
\hline & Liderazgo & 1 & 1.1 \\
\hline & Capacidad de adaptación & 1 & 1.1 \\
\hline & Motivación al estudio & 1 & 1.1 \\
\hline \multicolumn{2}{|l|}{ TOTAL } & 87 & 100 \\
\hline \multicolumn{4}{|c|}{ "Significado de Simulación Clínica" (SSC) } \\
\hline \multirow{6}{*}{ Escenario de aprendizaje } & Parecido a la realidad & 24 & 30.0 \\
\hline & Se aprende por ensayo y error & 6 & 7.5 \\
\hline & Aula universitaria & 1 & 1.3 \\
\hline & Delimitado & 1 & 1.3 \\
\hline & Controlado & 1 & 1.3 \\
\hline & Utiliza tiempo real & 1 & 1.3 \\
\hline \multirow{3}{*}{ Aprendizajes adquiridos } & Procedimentales & 9 & 11.2 \\
\hline & Administrativos & 7 & 8.7 \\
\hline & Fundamentos teóricos & 7 & 8.7 \\
\hline \multirow{4}{*}{ Supervisión Docente } & Asigna roles equipo multidisciplinario & 8 & 10.0 \\
\hline & Guía & 5 & 6.2 \\
\hline & Comparte y entrega conocimientos & 3 & 3.7 \\
\hline & Otorga realismo & 1 & 1.3 \\
\hline \multirow{2}{*}{ Evaluación } & Autoevaluación & 5 & 6.2 \\
\hline & Co-evaluación & 1 & 1.3 \\
\hline \multicolumn{2}{|l|}{ TOTAL } & 80 & 100 \\
\hline \multicolumn{4}{|c|}{ "Competencias genéricas y Simulación Clínica" (CGSC) } \\
\hline \multirow{4}{*}{ Interpersonales } & Trabajo en equipo & 13 & 31.0 \\
\hline & Trabajo en equipo interdisciplinar & 3 & 7.1 \\
\hline & Crítica y autocrítica & 2 & 4.8 \\
\hline & Habilidades interpersonales (respeto) & 1 & 2.4 \\
\hline \multirow{3}{*}{ Sistémicas } & Adaptarse a nuevas situaciones & 10 & 23.8 \\
\hline & Liderazgo & \begin{tabular}{|l|}
4 \\
\end{tabular} & 9.5 \\
\hline & Capacidad de aprender & 1 & 2.4 \\
\hline \multirow[b]{2}{*}{ Instrumental } & Conocimientos básicos de la profesión & 4 & 9.5 \\
\hline & Organizar y planificar & 3 & 7.1 \\
\hline
\end{tabular}




\begin{tabular}{|c|c|c|c|}
\hline & Resolución de problemas & 1 & 2.4 \\
\hline \multirow{2}{*}{\multicolumn{2}{|c|}{$\begin{array}{l}\text { TOTAL } \\
\text { "Factores que favorecen el aprendizaie con la Simulación Clínica" (FFSC) }\end{array}$}} & 42 & 100 \\
\hline & \multicolumn{3}{|c|}{ "Factores que favorecen el aprendizaje con la Simulación Clínica" (FFSC). } \\
\hline \multirow{5}{*}{$\begin{array}{l}\begin{array}{l}\text { Características } \\
\text { estudiantes }\end{array} \\
\text { de }\end{array}$} & Trabajo en equipo & 8 & 20.0 \\
\hline & Responsabilidad & 4 & 10.0 \\
\hline & Tener conocimientos & 4 & 10.0 \\
\hline & Imaginación & 4 & 10.0 \\
\hline & Buena disposición & 2 & 5.0 \\
\hline \multirow{6}{*}{ Aspectos de las/os Docente } & Enseñe procedimientos & 4 & 10.0 \\
\hline & Preocupación & 3 & 7.5 \\
\hline & Retroalimente & 3 & 7.5 \\
\hline & Con experiencia clínica & 2 & 5.0 \\
\hline & Con conocimientos teóricos & 2 & 5.0 \\
\hline & Creatividad & 2 & 5.0 \\
\hline Institucional & Disposición compra insumos & 2 & 5.0 \\
\hline \multicolumn{2}{|l|}{ TOTAL } & 40 & 100 \\
\hline \multicolumn{4}{|c|}{ "Proceso Enfermero y Simulación Clínica" (PESC) } \\
\hline \multirow{6}{*}{ Etapa Ejecución } & Reanimación cardiopulmonar básica & 10 & 25.6 \\
\hline & Precauciones estándar (manejo áreas/lavado manos) & 8 & 20.5 \\
\hline & Signos vitales & 3 & 7.7 \\
\hline & Toma de muestra & 2 & 5.1 \\
\hline & Uso incubadora & 3 & 7.7 \\
\hline & Instalación de vías (niño) & 1 & 2.6 \\
\hline \multirow{4}{*}{ Etapa Valoración } & Análisis de exámenes & 3 & 7.7 \\
\hline & Reconocimiento de signos y síntomas & 2 & 5.1 \\
\hline & Respeto por el paciente & 2 & 5.1 \\
\hline & Incluir a la familia & 1 & 2.6 \\
\hline Etapa Evaluación & Evolución del paciente & 4 & 10.3 \\
\hline \multicolumn{2}{|l|}{ TOTAL } & 39 & 100 \\
\hline \multicolumn{4}{|c|}{ "Factores que dificultan el aprendizaje con la Simulación Clínica" (FDSC). } \\
\hline \multirow{5}{*}{$\begin{array}{l}\begin{array}{l}\text { Características de } \\
\text { estudiantes }\end{array} \\
\text { los }\end{array}$} & Timidez & 6 & 18.7 \\
\hline & Falta de preparación previa & 3 & 9.3 \\
\hline & No les gusta la simulación & 2 & 6.3 \\
\hline & Estrés & 2 & 6.3 \\
\hline & Irritabilidad al asumir roles & 1 & 3.1 \\
\hline \multirow{5}{*}{ Institucional } & Fantomas no reales & 6 & 18.7 \\
\hline & Medicamentos y jeringuillas & 3 & 9.0 \\
\hline & Separar los ambientes hospitalarios & 2 & 6.3 \\
\hline & Centro de Simulación no terminado & 1 & 3.1 \\
\hline & Equipamiento no real (fototerapia) & 1 & 3.1 \\
\hline \multirow{2}{*}{ Organización actividad } & Elevado número de estudiantes por grupo & 2 & 6.3 \\
\hline & Sistema de rotación & 1 & 3.1 \\
\hline $\begin{array}{lll}\begin{array}{l}\text { Características } \\
\text { Docentes }\end{array} & \text { de } & \text { las/os } \\
\end{array}$ & Falta disposición & 2 & 6.3 \\
\hline \multicolumn{2}{|l|}{ TOTAL } & 32 & 100 \\
\hline
\end{tabular}

Fuente: Elaboración propia (2018)

Nivel 2: en este nivel emergieron tres núcleos temáticos o metacategorías que surgen de las seis categorías descriptivas codificadas del Nivel 1. Para ello se realizó un proceso de comparación intercategorías en el que se buscaron similitudes estructurales y elementos 
comunes de este análisis, emergiendo tres núcleos temáticos o metacategorías que representan la realidad estudiada tal como lo describen los informantes claves.

1. Experiencia de los estudiantes de la Carrera de Enfermería en relación a la Simulación Clínica: percepción en torno al concepto y experiencia como estrategia de aprendizaje en su formación profesional en la Carrera de Enfermería (SSEX SSC).

2. Factores que facilitan y obstaculizan el aprendizaje con la Simulación Clínica: apreciación en relación a aspectos que contribuyen y dificultan el aprendizaje, relacionados a aspectos de los estudiantes, docentes, insumos, infraestructura y organización de la actividad (FFSC - FDSC).

3. Competencias desarrolladas en estudiante de enfermería, a través de la Simulación Clínica: opiniones vinculadas a las competencias específicas dentro del proceso enfermero y genéricas (instrumentales, sistémicas e interpersonales) (PESC CGSC).

Nivel 3: de lo anterior surgen dos dominios cualitativos:

1. Aportes de los estudiantes de enfermería para implementar la Simulación Clínica: representa las opiniones en relación a la vivencia que les permitió conceptualizarla e identificar factores concurrentes en su aplicación (SEX - SSC - FFSC - FDSC).

2. Contribución de la Simulación Clínica como estrategia de aprendizaje para el desarrollo de competencias disciplinares y genéricas para su futuro desempeño profesional: evidencia la valoración con respecto al desarrollo del proceso enfermero como competencias disciplinares y las genéricas (instrumentales, sistémicas e interpersonales) (PESC - CGSC).

\section{DISCUSIÓN Y CONSIDERACIONES FINALES}

En el Nivel 1, las unidades de significado para las dos primeras categorías "Significado de haber vivido esta experiencia de aprendizaje" y "Significado de Simulación Clínica" con un $27,2 \%$ y un $25 \%$ respectivamente, refleja lo relevante del tema dentro de la formación de los informantes claves. La primera evidencia, al igual que en otros estudios, dos grandes aspectos: uno profesional (adquisición de conocimiento, técnicas, procedimientos y las funciones de administración/gestión y educación y otro personal) $(4,5,22)$, y otro personal -consecución de competencias genéricas- $(5,20,21,23)$. El segundo coincide con lo expuesto en la literatura, declarando que son escenarios de aprendizaje en los cuales se adquiere el conocimiento supervisado por un docente y donde se aplica la evaluación tanto de proceso como resultado $(4,5,8)$. Lo expresado refleja lo significativo que es trabajar con esta estrategia de aprendizaje, ya que sin tener una clase teórica de Simulación Clínica la lograron definir sólo con el desarrollo de ésta.

En relación a "Competencias genéricas y Simulación Clínica", lo encontrado se clasificó de acuerdo al Tunning (1) en Interpersonales, Sistémicas e Instrumental y al identificarlas en cada una de ellas, se observa que sólo el trabajo en equipo es asimilable con otro estudio (5). Competencias como autonomía (19), autoeficacia (20), comunicación (21) y reflexión (22) no emergen en los discursos; sin embargo sí identifican respeto, adaptarse a nuevas situaciones, liderazgo, capacidad de aprender, 
organizar, planificar y resolución de problemas, lo que podría deberse a que en los estudios mencionados se utilizó otro tipo de clasificación de competencias genéricas. No menos cierto, habría que considerar que los estudiantes fueron sometidos a una situación de aprendizaje y proceso de evaluación nuevo. De todas formas, lo encontrado es relevante ya que las actuales directrices educacionales apuntan a fortalecer estas cualidades como un factor del capital humano (2).

Con respecto al "Proceso Enfermero y Simulación Clínica", se identifican tres etapas, valoración, ejecución y evaluación, siendo reconocida mayormente la ejecución; las no declaradas, diagnóstico de necesidades y planificación, probablemente se debe al nivel de dificultad (bajo) de los escenarios de la simulación clínica, tomado en consideración por los docentes al momento de efectuar esta primera experiencia como una forma de ir ensayando la metodología $(3,15)$. Que los informantes clave consideren la aplicación del Proceso Enfermero, reafirma que en el nivel curricular que se encuentran tienen claridad de lo que pueden integrar en relación a los grados de dificultad que se les presentan para su aprendizaje.

En cuanto a la categoría "Factores que favorecen el aprendizaje con la Simulación Clínica”, emergen tres aspectos; características de los estudiantes, de las/os docentes e Institución, reconociendo al primero de ellos como el más relevante con 22 unidades de significado, destacando el trabajo en equipo, muy importante como competencia genérica. Lo que emerge sobre las opiniones de las/os docentes se enmarca en lo descrito por Quiroz y Vargas, quienes sostienen que deben estar capacitados en aspectos pedagógicos pertinentes y ser preocupados y creativos en el momento de realizar la acción educativa (4). En lo referente a proporcionar insumos por parte de la Institución señalan sólo dos unidades de significado en el mismo sentido que lo planteado por Juguera, Díaz, Pérez et al., con respecto a simuladores más cercanos a la realidad (5).

Lo referente a la categoría "Factores que dificultan el aprendizaje", se relacionan principalmente a las características personales de los estudiantes, las que podrían disminuir si esta metodología se incorporara en forma paulatina a lo largo de la carrera (20). En cuanto al factor Institucional, los informantes se refieren a insumos insuficientes y fantomas no reales e infraestructura no apropiada para estos efectos, lo que es concordante con el estudio de Juguera, Díaz, Pérez et al., donde los estudiantes sugirieron una mejora en la adquisición de material real y menos simulado (5). Así mismo, encuentran que en la organización de la actividad se debería disminuir el número de estudiantes para facilitar el proceso planificando y organizarlo de acuerdo a la creciente complejidad del plan de estudio y a protocolos que garanticen la reproducción de escenarios, de forma que se vaya instaurando una cultura de la simulación clínica considerando además la colaboración entre docentes y estudiantes $(4,19,25)$.

Finalmente, señalar que la información obtenida en este estudio, concuerda con lo mostrado por varios autores antes mencionados, en el sentido que es una estrategia relevante a la hora de integrar conocimientos y destrezas, que la experiencia previa es un aspecto importante a la hora de ejecutar esta metodología, reduce la brecha existente entre la teoría y práctica, fortalece las competencias genéricas y permite que el estudiante asuma la responsabilidad en su formación 


\section{REFERENCIAS BIBLIOGRÁFICAS}

1. Tuning Educational Structures in Europe. Informe Final Proyecto Piloto-Fase 1. Bilbao: Edición y Cultura. Sócrates. 2003.

2. Barrios S, Masalán P, Cook MP. Educación en Salud: en la búsqueda de metodologías innovadoras. Cienc. Enferm. 2011; 17(1): 57-69.

3. Alfaro-Lefevre R. Aplicación del proceso enfermero: fomentar el cuidado en colaboración. Quinta Edición. México: Masson. 2003.

4. Quirós S, Vargas A. Simulación clínica: una estrategia que articula prácticas de enseñanza e investigación en Enfermería. Texto \& Contexto - Enferm. 2014; 23 (4): 815-816.

5. Juguera L, Díaz J, Pérez $M^{a}$ L, Leal C, Rojo A, Echevarría P. La simulación clínica como herramienta pedagógica: percepción de los alumnos de Grado en Enfermería en la UCAM (Universidad Católica San Antonio de Murcia). Enfermería Global. 2014; 13 (33): 175-190.

6. Ministerio de Salud de Chile (MINSAL, 2016). Ley de Derechos y Deberes del Paciente No20.584 [citado 2019 may. 15]. Disponible en: http://www.ssmso.cl/tmpArchivos/participacion/Escuelas/Leyes/Ley\%20de\%20Dere chos $\% 20 y \% 20$ Deberes $\% 20 \mathrm{de} \% 201$ os $\% 20$ pacientes $\% 20$ Superintendencia $\% 20 \mathrm{de} \% 20$ Salud.pdf

7. Galindo J, Visbal L. Simulación, herramienta para la educación médica Simulation, a teaching aid for medical education. Salud Uninorte. Barranquilla (Col.). 2007: 23(1): 79-95.

8. Bland A, Topping A, Wood B. A concept analysis of simulation as a learning strategy in the education of undergraduate nursing students. Nurse Education Today. 2011; 31(7): 664-670.

9. Del Moral I, Díaz de Terán J, Rabanal J, Quesada A, Rodríguez J, Teja J, et al. Nuevos procedimientos de entrenamiento en el manejo de crisis y emergencias médicas. En Procedimientos técnicos en urgencias y emergencias. Editores Quesada A, Rabanal JM, editores. Madrid 2003 pp; 479-86.

10. De la Horra I. La simulación clínica como herramienta de evaluación de competencias en la formación de enfermería. Reduca (Enfermería, Fisioterapia y Podología). Serie Trabajos Fin de Máster. 2010; 2(1): 549-580.

11. Ziv A. Simulators and simulation-based medical education, en Dent, J. y Harden, R.M. (eds.) A Practical Guide for Medical Teachers, Edinburgh; 2009. P 217-222.

12. Ausubel D, Novak J, Hanesian H. Psicología educativa. Un punto de vista cognitivo. Segunda Edición. México: Trillas; 2000.

13. Dewey J. Experiencia y educación Libro de John. Estados Unidos: Kappa Delta Pi; 1938.

14. Ruiz M. Enfoque integral del currículo para la formación de profesionales competentes. México: Instituto Politécnico Nacional; 2000. 
15. Vázquez-Mata G, Guillamet-Lloveras A. El entrenamiento basado en la simulación como innovación imprescindible en la formación médica. Educ Med. 2009;12(3): 149-155.

16. Velazco-Martín A. Simulación Clínica y enfermería creando un ambiente de simulación [Tesis de Grado]. Universidad de Cantabria. Disponible en https://repositorio.unican.es/xmlui/bitstream/handle/10902/3949/VelascoMartinA.pd f. 2013.

17. Maestre J, Manuel-Palazuelos J, del Moral I, Simond Robert. La simulación clínica como herramienta para facilitar el cambio de cultura en las organizaciones de salud: aplicación práctica de la teoría avanzada del aprendizaje. Rev colomb. Anestesiol. 2014; 42(2): 124-128.

18. Maestre J, Rudolph J. Teorías y estilos de debriefing: el método con buen juicio como herramienta de evaluación formativa en salud. Rev Esp Cardiol. 2015;68(4): 282-5. DOI: 10.1016/j.recesp.2014.05.018

19. Niño CA, Vargas NG, Barragán JA. Fortalecimiento de la simulación clínica como herramienta pedagógica en enfermería: experiencia de internado. Rev Cuid. 2015; 6(1): 970-5. http://dx.doi.org/10.15649/cuidarte.v6i1.161

20. Barrios S, Urrutia M, Rubio M. Impacto de la simulación en el desarrollo de la autoeficacia y del locus de control en estudiantes de enfermería. Educación Médica Superior. 2017; 31(1):125-136.

21. Ching-Lan E, Shiah-Lian Ch, Shu-Yuan Ch, Yueh-Chih Ch. Using standardized patient with immediate feedback and group discussion to teach interpersonal and communication skills to advanced practice nursing students. Nurse Education Today. 2013; 33(6): 677-683.

22. Johannesson E, Silén Ch, Kvist J, Hult H. Students' experiences of learning manual clinical skills through simulation. Adv in Health Sci Educ. 2013; 18(1):99-114.

23. Tagwa O. Nursing Students' Perceptions of Satisfaction and Self-Confidence with Clinical Simulation Experience. Journal of Education and Practice. 2016; 7(5): 131-138.

24. Ruiz S. Simulación Clínica y su utilidad en la mejora de la seguridad de los pacientes. Trabajo Fin de Grado. 2012. Departamento de Enfermería, Universidad Cantabria. Disponible en http://www.asepur.org/wp-content/uploads/2014/06/Simulacion-Clinica-y-su-utilida d-en-la-mejora-de-la-seguridad-de-los-pacientes.pdf

25. Zhang J. Perceptions of simulation-assisted teaching among baccalaureate nursing students in Chinese context: Benefits, process and barriers. Journal of Professional Nursing. 2017; 33(4): 305-310.

26. Tas F, Sonay A, Celen R, Özkan S, Altıparmak D, Sahin A. Comparing Traditional and Simulation-Based Experiences in Pediatrics with Undergraduate Nursing Students in Turkey. Clinical Simulation in Nursing. 2018; 16: 62-69. 
27. Lubbers J, Rossman C. The effects of pediatric community simulation experience on the self-confidence and satisfaction of baccalaureate nursing students: A quasi-experimental study. Nurse Educ Today. 2016; 39: 93-98.

28. Latorre A, Del Rincón D, Arnal J. Bases metodológicas de la investigación educativa. Barcelona: Hurtado; 1999.

29. Polit D, Hungler BP. Investigación Científica en Ciencias de la Salud. Sexta Edición. México: Mc Graw- Hill; 2000.

30. Martínez-Salgado C. El muestreo en investigación cualitativa: principios básicos y algunas controversias. Ciênc. Saúde Coletiva. 2012; 17 (3): 613-9. http://doi.org/b9qn.

31. Glaser BG, Strauss AL. El método de comparación constante de análisis cualitativo. In The discovery of Grounded Theory: strategies for qualitative research New York: Aldine; 1967. P 45-77.

32. Gil-Flores J, Perera-Rodríguez VH. Análisis informatizado de datos cualitativos. Sevilla: Kronos; 2001.

33. Miles MB, Huberman AM. Qualitative data analysis: A sourcebook of new methods. Beverly Hills: Sage; 1984.

34. Latorre A. La investigación-acción: conocer y cambiar la práctica educativa. Barcelona: Graó; 2003.

35. Guba EG, Lincoln YS. Naturalist Inquiry. London: Sage; 1985.

36. Universidad Autónoma de Chile. Acta de Evaluación Nº 0131-16. 2016.

37. Lolas F, Quezada A, (eds.). Pautas éticas de investigación en sujetos humanos: nuevas perspectivas. Chile: Programa Regional de Bioética OPS/OMS; 2003.

El trabajo se enmarca en el Programa de Magíster Innovación de la Docencia Universitaria en Ciencia de la Salud. Facultad de Medicina, Universidad de La Frontera. 